\title{
Using Unscented Kalman Filter in Subsurface Contaminant Transport Models
}

\author{
S. Y. Chang ${ }^{1}$ and M. Sayemuzzaman ${ }^{2, *}$ \\ ${ }^{I}$ Department of Civil and Environmental Engineering, North Carolina A\&T State University, Greensboro NC 27411, USA \\ ${ }^{2}$ Department of Energy and Environmental System, North Carolina A\&T State University, Greensboro NC 27411, USA
}

Received 9 March 2012; revised 22 October 2012; accepted 18 March 2013; published online 21 March 2014

\begin{abstract}
Using a traditional numerical approach in subsurface contaminant transport models generates unavoidable deviation due to unknown or uncertain sources, inaccurate transport and hydraulic parameters, and numeric scheme errors. As a result, stochastic data assimilation or filtering techniques have been employed in the subsurface simulation processes to improve the accuracy of model results. The Kalman Filter (KF) has been widely used for estimation and tracking of linear systems. In the subsurface transport model, even if the system dynamics are linear, it can become a nonlinear one because of the presence of the unknown parameters. The Unscented Kalman Filter (UKF) is one of the data assimilation filters that offer a potential solution to the problem of model development with noisy and incomplete data when the system is nonlinear. The objective of this study was to apply the UKF in subsurface contaminant transport models and to find the contaminant plume. The performance was then evaluated in comparison with the KF and numerical model. A two dimensional transport model with advection and dispersion was used as the deterministic model of a conservative contaminant transport in the subsurface. Random Gaussian noises were added to the numerical method result to simulate the true solution and the observation data. Then the UKF and KF filtering techniques were applied for the data assimilation. An Error Standard Deviation (ESD) of pollutant concentrations was used to examine the effectiveness. The UKF can reduce $6 \sim 75 \%$ and $2 \sim 52 \%$ of prediction errors when compared with the numerical and KF results, respectively.
\end{abstract}

Keywords: stochastic process; Error Standard Deviation (ESD); Kalman Filter (KF); Unscented Kalman Filter (UKF); contaminant transport

\section{Introduction}

The prevention and control of groundwater contamination can be enhanced if the sources of contamination, types of contaminants, and the movement of contaminants through porous media are taken into consideration. Subsurface contaminant transport models play an important role to groundwater risk and clean-up processes. One of the main goals of subsurface contaminant transport modeling is to predict the contaminant concentration with limited parametric information. Subsurface contaminant transport models can be one, two, or three dimensional. Mathematical deterministic models (Goovaerts, 1999; Kim and Parizek, 1999; Ren and Zhang, 1999; Parkin et al., 2007) are widely used in subsurface contaminant transport processes. However, a numerical method suffers, with its numerical errors including the truncation error (TE) and convergence error. When these numerical methods are used in the subsurface transport model, it provides deviated predictions due to the inherent randomness and uncertainty in the transport system. Two major factors contribute to these uncertainties. Firstly,

\footnotetext{
" Corresponding author. +1 336 6862884; fax: +1 3362562344 .

E-mail address: msayemuz@ncat.edu (M. Sayemuzzaman).
}

ISSN: 1726-2135 print/1684-8799 online

(C) 2014 ISEIS All rights reserved. doi: 10.3808/jei.201400253 field observations provide the environmental model parameters and data. Unavoidable variability of hydraulic properties exists on those field observations due to the heterogeneous subsurface flow (Heuvelink and Webster, 2001; Zheng and Bennett, 2002; Hu et al., 2009). Secondly, these uncertainties are also caused by errors in model mechanisms and numerical schemes. By incorporating the random field with the deterministic numerical model, the stochastic dynamic system of transport process can be represented (Hamed et al., 1996; Van et al., 1999; Pham, 2001; Chan and Govindaraju, 2006; Saad, 2007; Zhang et al., 2009). Furthermore, the deterministic system model cannot work well alone. Thus the system model updated by observations can provide better solution than those by a traditional deterministic model. Thus, the stochastic data assimilation filtering technique can be used to overcome the traditional deterministic model problems.

The Kalman Filter (KF) has been widely used for estimation and tracking of linear systems due to its simplicity and robustness (Grewal and Andrews, 1993; Schreider et al., 2001). Most of the contaminant transport models possess some degree of nonlinearity. The degree of nonlinearity depends on the type of assumption and the relevant equations used to solve the model (Magnus et al., 2000; Li and Zhang, 2010). In most cases, the linear approximation may not be accurate enough and nonlinear process models are necessary for the state estimation (Creveling, 2008). The most common way of applying the KF 
to a nonlinear system is in the form of the Extended Kalman Filter (EKF). In the EKF, the PDF (Probability Density Function) is propagated through a linear approximation of the system at each time step. In doing so, the EKF needs the Jacobian matrices which may be difficult to obtain for higher order systems, especially in the case of time-critical applications (Costa, 1994; Pastres et al., 2003). In order to overcome the drawbacks of the EKF, other nonlinear state estimators have been developed such as the Unscented Kalman Filter (UKF) (Julier and Uhlmann, 1997, 2004). The UKF method yields a filter that is more accurate than an EKF and easier to implement than an EKF or a Gauss second-order filter (Simon et al., 2000; Wan and Rudolph, 2000). UKF and KF are state space models that can be used as a dynamic technique. The state space model is a method for analyzing a wide range of time series models. When the time series is represented by the state space model (SSM), the KF and UKF are used for filtering, prediction, and smoothing of the state vector. Thus, they can assimilate new measurement (information) dynamically. The objective of this paper is to apply the UKF in subsurface contaminant transport modeling and to find the contaminant plume. The performance is then evaluated in comparison with the $\mathrm{KF}$ and deterministic numerical model.

\section{Methodology}

\subsection{Advection-Dispersion-Adsorption Model}

A two dimensional transport model with advection and dispersion was used as the deterministic model of a conservative contaminant transport in the subsurface. The advectiondispersion model in subsurface describes contaminant transport in the groundwater by means of partial differential equations derived from solute mass balances. In this study, a twodimensional subsurface contaminant model in the horizontal plane $(\mathrm{x}-\mathrm{y})$ and advection in the $\mathrm{x}$ direction was used to examine the effectiveness and accuracy of the numerical KF and UKF results relative to the true solution. In the model, sorption is also considered as an important factor. The advection-dispersion-adsorption model is represented by the following equation:

$\frac{\partial C}{\partial t}=\frac{D_{x}}{R} \frac{\partial^{2} C}{\partial x^{2}}+\frac{D_{y}}{R} \frac{\partial^{2} C}{\partial y^{2}}-\frac{V}{R} \frac{\partial C}{\partial x}$

Here $\mathrm{R}$ is the retardation factor which is defined by:

$R=1+\rho\left(\frac{k_{d}}{\varphi}\right)$

where $\mathrm{C}=$ contaminant concentration $(\mathrm{mg} / \mathrm{l}) ; \mathrm{t}=$ time $(\mathrm{s}), D_{x}$, $D_{y}=$ dispersion coefficients in the $x$ and $y$ direction, respectively $\left(\mathrm{m}^{2} / \mathrm{s}\right) ; x, y=$ cartesian space coordinates $(\mathrm{m}) ; V=$ the average linear pore liquid velocity $(\mathrm{m} / \mathrm{s}) ; \mathrm{R}=$ retardation factor, dimensionless; $\rho=$ dry bulk density of the soil $(\mathrm{mg} / \mathrm{l}) ; k_{d}=$ distribution coefficient for the solute in the soil $(1 / \mathrm{mg}) ; \varphi=$ volumetric moisture content of the soil, dimensionless.
In this study, an initial spontaneous point mass source at $\left(x_{0}, y_{0}\right)$ and a constant boundary condition has been taken. The boundary condition of the two dimensional mass transport with an instantaneous point source is expressed as:

$$
\left.C(x, y, t)\right|_{t=0}=C_{I}\left(x_{0}, y_{0}\right) \text { and }\left.C(x, y, t)\right|_{\Omega}=C_{B}
$$

where $C_{B}$ is constant concentration at boundary $\Omega$, and $\Omega$ is chosen as a square boundary in this study.

\subsection{State-Space Formulation of the Contaminant Transport Modeling}

In order to solve the two-dimensional advection-dispersion-adsorption equation numerically, a Forward-Time and Central-Space (FTCS) differencing scheme was developed. Zou and Parr (1995) used this explicit finite-difference method (FDM) in their research to predict contaminant transportation in a two-dimensional aquifer condition. Similarly, Chang and Jin (2005) proposed the use of FTCS in their contaminant modeling approach using the Kalman Filter. In this paper, the same numerical approach is used to generate the deterministic numerical model. Gaussian random noises were used with this numerical solution to generate the true solution and accompanying observation. That observation was used to guide the filtering process. A MATLAB program was developed to solve the numerical model explicitly and to estimate the concentration of the conservative contaminant spatially and temporally. After solving Equation (1) by using FTCS, the general form of the Equation (1) can be rewritten in a state-space form as:

$\mathbf{x}_{(t+1)}=\mathbf{A} * \mathbf{x}_{(t)}$

where $\mathbf{x}_{(t+1)}$ is the vector of contaminant concentration at all nodes at time $\mathrm{t}+1 ; \mathbf{x}_{(\mathrm{t})}$ is the vector of contaminant concentration at all nodes at time $t ; \mathbf{A}$ is the State Transition Matrix (STM) containing the parameters for the model.

The convergence and stability criteria for the scheme are given as:

$\Delta x<\frac{2 D_{x}}{V}$ and $\Delta t<\frac{\Delta x^{2} R}{2 D x}$

where, $\Delta x, \Delta y$ and $\Delta t$ are the discretized space in $x$ and $y$ direction and time, respectively. Capitalized bold letter denotes matrices and small bold letter denotes vectors.

\section{Stochastic Process}

Stochastic processes are introduced for modeling the complicated real-world transport systems which usually have unknown or uncertain sources, inaccurate transport parameters, or numeric errors. The stochastic dynamics can be represented as deterministic dynamics of transport and a random noise term (Pham, 2001; Saad, 2007).It can be written as: 
$\mathbf{x}_{\mathrm{t}+1}=\mathbf{A} \mathbf{x}_{\mathrm{t}}+\mathbf{p}_{\mathrm{t}}, \mathrm{t}=0,1,2,3, \ldots, \mathrm{t}_{\mathrm{n}}$

where $\mathbf{x}_{\mathrm{t}}$ and $\mathbf{x}_{\mathrm{t}+1}$ are the subsurface state variables at time $t$ and $t+1$, respectively. $\mathbf{A}$ is the finite-difference operator that advances the $\mathbf{x}_{t}$ by one time step. $\mathbf{p}_{t}$ is the model system error, the difference between the model prediction and the optimal estimate of the true value. The model error vector $\mathbf{p}_{\mathrm{t}}$ is assumed to have covariance matrix $\mathbf{Q}_{t}$ and zero mean.

Observation $\mathbf{z}_{\mathrm{t}}$ are described by data conversion matrix $\mathbf{H}$ and error $\mathbf{o}_{\mathrm{t}}$. Similarly, $\mathbf{o}_{\mathrm{t}}$ is assumed to be the error of observations that has covariance matrix $\mathbf{R}_{t}$ and zero mean:

$\mathbf{z}_{\mathrm{t}}=\mathbf{H} \mathbf{x}_{\mathrm{t}}^{\mathrm{T}}+\mathbf{o}_{\mathrm{t}}$

\subsection{Data Assimilation with Kalman Filtering (KF)}

Considering a stochastic process defined by Equations (6) and (7) above, the Kalman optimal estimator (Grewal and Andrews, 1993 ; Schreider et al., 2001) is:

$\mathbf{x}_{\mathrm{t}+1}(+)=\mathbf{x}_{\mathrm{t}+1}(-)+\mathbf{K}_{\mathrm{t}+1}\left[\mathbf{z}_{\mathrm{t}+1}-\mathbf{H} \mathbf{x}_{\mathrm{t}+1}(-)\right]$

where $\mathbf{x}_{\mathrm{t}+1}(+)$ indicates the estimated value after the KF adjustment, and $\mathbf{x}_{t+1}(-)$ the value before the KF adjustment, i.e. the predicted value from the model. The matrix $\mathbf{K}_{\mathrm{t}+1}$ is determined by:

$\mathbf{K}_{\mathrm{t}+1}(+)=\mathbf{P}_{\mathrm{t}+1}(-) \mathbf{H}^{\mathrm{T}}\left(\mathbf{H} \mathbf{P}_{\mathrm{t}+1}(-) \mathbf{H}^{\mathrm{T}}+\mathbf{R}_{\mathrm{t}+1}\right)^{-1}$

where $\mathbf{P}_{t+1}$ is the optimal estimate error covariance matrix. ( ) $)^{\mathrm{T}}$ and ()$^{-1}$ denote the transpose and inverse of matrix, respectively. The $\mathbf{P}_{\mathrm{t}+1}$ can be obtained by the following recursive calcula- tion:

$\mathbf{P}_{\mathrm{t}+1}(+)=\mathbf{P}_{\mathrm{t}+1}(-)-\mathbf{P}_{\mathrm{t}+1}(-) \mathbf{H}^{\mathrm{T}}\left[\left(\mathbf{H} \mathbf{P}_{\mathrm{t}+1}(-) \mathbf{H}^{\mathrm{T}}+\mathbf{R}\right]^{-1} \mathbf{H} \mathbf{P}_{\mathrm{t}+1}(-)\right.$

$\mathbf{P}_{\mathrm{t}+1}(-)=\mathbf{A} \mathbf{P}_{\mathrm{t}}(+) \mathbf{A}^{\mathrm{T}}+\mathbf{Q}_{\mathrm{t}}$

As seen in Equation (8), $\mathbf{K}_{\mathrm{t}+1}$ determines how much the estimated value can gain from the observations; therefore, $\mathbf{K}_{t+1}$ in Equqation (9) is called the Kalman optimal gain matrix or Kalman Filter. In addition to advancing a time sequence, the system operator A also simultaneously applies to a space sequence, $\mathbf{x}[i], i=0,1,2, \ldots, n$ where index $i$ indicates the space positions and $\mathrm{n}$ is the total spaces. Therefore, the KF has the potential for assimilating data in both space and time. Accordingly, the observational data pattern matrix $\mathbf{H}$ can represent the incomplete and irregular pattern of observations in both space and time. Note that KF can be conveniently implemented since the model dynamic system itself has already been integrated in the assimilation (Grewal and Andrews, 1993; Jin, 1996; Chang and Jin, 2005).

\subsection{Optimal Estimation and Data Assimilation with Unscented Kalman Filter}

The Unscented Kalman Filter (UKF) was developed to address the deficiencies of the linearization of the Extended Kalman Filter (EKF) by providing a more direct and explicit mechanism for transforming the mean and covariance information (Rambabu et al., 2008). The UKF is founded on the premise that it is easier to approximate a probability distribution than an arbitrary nonlinear function or transformation (Simon et al., 2000). Although the UKF method superficially resembles the Monte Carlo method and the particle filter method, it differs in that the samples in the UKF are not drawn at random. Rather, the samples are deterministically chosen so that they can capture specific information about the distribution. As a result, high-order information about the distribution can be captured with a fixed, small number of points. In general, this scheme can be applied to capture many kinds of information about many types of distributions (Julier and Uhlmann, 1997, 2004; Rudolph and Eric, 2001; Gove and Hollinger, 2006).

Let $\mathrm{n}_{\mathrm{x}}$ be the dimension of state vector $\mathbf{x}$ with mean $\overline{\mathbf{x}}$ and covariance $\mathrm{P}_{\mathrm{x}}$. A set of sigma points $\boldsymbol{\sigma}$ consists of $2 \mathrm{n}_{\mathrm{x}}+1$ vectors and associated weights $\mathrm{S}=\left\{\sigma_{i}, W_{i} ; i=0,1,2, \ldots, 2 n_{\mathrm{x}}\right\}$. The weights $W_{i}$ can be positive or negative but, to provide an unbiased estimate, they must obey the condition:

$\sum_{i=0}^{2 \mathrm{n}_{\mathrm{x}}} W_{i}=1$

The selection scheme for sigma points and weights are:

$$
\begin{aligned}
& \sigma_{i}=\bar{x}, i=0 \\
& \sigma_{i}=\bar{x}+\left(\sqrt{\left(n_{x}+\lambda\right) P_{x}}\right)_{i}, i=1,2, \ldots, \mathrm{n}_{\mathrm{x}} \\
& \sigma_{i}=\bar{x}-\left(\sqrt{\left(n_{x}+\lambda\right) P_{x}}\right)_{i}, i=\mathrm{n}_{\mathrm{x}}+1, \ldots, 2 \mathrm{n}_{\mathrm{x}} \\
& W_{0}^{(m)}=\frac{\lambda}{\lambda+n_{x}}, i=0 \\
& W_{0}^{(c)}=\frac{\lambda}{\lambda+n_{x}}+\left(1-\alpha^{2}+\beta\right), i=0 \\
& W_{i}^{(m)}=W_{i}^{(c)}=\frac{1}{2\left(\lambda+n_{x}\right)}, i=\mathrm{n}_{\mathrm{x}}+1, \ldots, 2 \mathrm{n}_{\mathrm{x}}
\end{aligned}
$$

where $\lambda=\alpha^{2}\left(n_{x}+\kappa\right)-n_{x}$ the scaling parameters $\lambda, \alpha$ and $\beta$ are tuning parameters. Choosing $\kappa(\kappa \geq 0)$ guarantees positive semi-definiteness of the covariance matrix; a common default is $\kappa=0$. If $\kappa$ is negative there is the possibility, as with all approximation algorithms, that the predicted covariance will be non-postitive semi-definite. The range of the values of $\alpha$ is $10^{-4} \leq \alpha \leq 1$, which controls the spread of the sigma points and 
should ideally be a small number to avoid non-local effects when the nonlinearities are strong. $\beta$ is a non-negative weight which can be used to incorporate knowledge of the higher order moments of the distribution. For the Gaussian case, the optimal choice is $\beta=2$ (Cheryl and Juergen 2008). $\mathrm{W}_{i}^{(\mathrm{m})}$ and $\mathrm{W}_{i}^{(\mathrm{c})}$ represent the weight components to calculate the mean and covariance, respectively. The UKF state update and measurements update equations are stated below (Andrei and José, 2004).

\subsubsection{Time Update Equations}

Propagate each sigma point through the update function:

$Y_{i, t \mid t-1}=g\left(\sigma_{i, t-1}, u_{i, t-1}\right) ; i=0,1,2, \ldots, 2 n_{\mathrm{x}}$

The mean, covariance and the cross covariance are calculated as follows:

$$
\begin{aligned}
& \bar{y}(-) \approx \sum_{i=0}^{2 n_{x}} W_{i}^{(m)} Y_{i} \\
& P_{y_{t}}(-) \approx \sum_{i=0}^{2 n_{x}} W_{i}^{(\mathrm{c})}\left(Y_{i, t \mid t-1}-\bar{y}(-)\right)\left(Y_{i, t \mid t-1}-\bar{y}(-)\right)^{T}+R_{u} \\
& P_{x_{t} y_{t}} \approx \sum_{i=0}^{2 n_{x}} W_{i}^{(\mathrm{c})}\left(\sigma_{i}-\bar{x}\right)\left(Y_{i}-\bar{y}\right)^{T}+R_{u}
\end{aligned}
$$

where $u$ is the system noise and $\mathrm{R}_{\mathrm{u}}$ is the system noise covariance matrix.

\subsubsection{Measurement Update Equations}

Transform the observation values through the measurement update equations:

$$
Z_{i,|t| t-1}=f\left(O_{i, t \mid t-1}, v_{i, t-1}\right) ; i=0,1,2, \ldots, 2 n_{\mathrm{x}}
$$

and the mean, covariance and cross-covariance of the measurement are calculated as follows:

$$
\begin{aligned}
& \bar{z}(-)=\sum_{i=0}^{2 \mathrm{n}_{\mathrm{x}}} W_{i}^{m} Z_{i, t \mid t-1} \\
& P_{z_{t}}(-) \approx \sum_{i=0}^{2 \mathrm{n}_{\mathrm{x}}} W_{i}^{(c)}\left(Z_{i, t \mid t-1}-\bar{z}(-)\right)\left(Z_{i, t \mid t-1}-\bar{z}(-)\right)^{T}+R_{v} \\
& P_{y_{t} z_{t}} \approx \sum_{i=0}^{2 \mathrm{n}_{\mathrm{x}}} W_{i}^{(c)}\left(Y_{i, t \mid t-1}-\bar{y}(-)\right)\left(Z_{i, t \mid t-1}-\bar{z}(-)\right)^{T}+R_{v}
\end{aligned}
$$

where $\mathrm{O}$ is the observation, $v$ is the measurement noise and $R_{v}$ is the measurement noise covariance matrix

The Unscented Kalman gain (U) is given by:
$U_{t}=P_{y_{t} z_{t}} P_{z, t}^{-1}$

and the UKF state estimation and its covariance are computed from the standard Kalman update equations:

$$
\begin{aligned}
& \bar{y}_{t}(+)=\bar{y}(-)+U_{t}\left(\bar{z}_{t}-\bar{z}_{t}(-)\right) \\
& P_{y_{t}}(+)=P_{y_{t}}(-)+U_{t} * P_{z_{t}}(-) * U_{t}^{T}
\end{aligned}
$$

\section{Case Study}

\subsection{Components of Parametric Input}

The system parameters used in this paper are based on the work of Zou and Parr (1995) and several research works previously conducted on the subsurface cotaminant transport model. In this study, the model area in the two-dimensional plane has 400 grid points with grid intervals of, $\Delta x=1.5$ and $\Delta y=1.5 \mathrm{~m}$. Therefore the model area is $30 \times 30 \mathrm{~m}^{2}$. The time interval per each time step $\Delta t=0.20$ day with a total time step of 50. Therefore, the total simulated period is 10 days. $\mathrm{V}$ and $\mathrm{R}$ are assumed to be $2.10 \mathrm{~m} / \mathrm{d}$ and 1.525 , respectively. The numerical solution was generated with different velocity than the true solution. Dx and Dy are 1.554 and $0.4662 \mathrm{~m}^{2} / \mathrm{d}$, respectively. This model is developed to deal with a conservative contaminant; hence the chemical decay term is eliminated. An instantaneous initial concentration of $1000 \mathrm{mg} / \mathrm{l}$ of any kind of conservative contaminant is injected into the grid point at coordinate $(5,10)$. In this research, a time-independent Gaussian system error having a standard deviation of $10 \%$ is injected into the numerical model to generate the process or system model, a 5\% Gaussian system error is also injected into the numerical model to generate the measurement or observation model.

\subsection{Generation of the True Solution}

In this study an artificial uncertainty system is created as the true solution, $T_{F}$. The true solution is simulated by using the numerical solution with injected random noises. In addition to that noise, a very small percentage of random noises is injected cumulatively with respect to the time step. The true solution also differed by generating a different State Transition Matrix (STM) than in the numerical solution [Equation (4)]. The true solution used $\mathrm{A}_{t}$ as a STM. Due to the random error injection to the system model and its operational pattern, the matrix $A_{t}$ varies with each time step. The average pore liquid velocity, $\mathrm{V}$ [Equation (1)] used is $1.5 \mathrm{~m} /$ day, which differs from the velocity used in the numerical solution. The numerical solution has no information about the changed velocity used in the true solution. In this study, an arbitrary $40 \%(2.10 \mathrm{~m} / \mathrm{d})$ higher velocity was used in the numerical model. As the transport process is being simulated, the UKF and KF are not informed of the existence of $T_{F}$, except in the limited observations that are extracted from the $T_{F}$. The simulation results provided by the $\mathrm{UKF}$ and KF can then be compared with the $T_{F}$ for examination. 


\subsection{Generation of Observation Data}

The state-space model works with limited system parameters like contaminant velocity, porosity of soil media, retardation factor, dispersion etc. These parameters may not be accurate enough to predict the true behavior of pollutants. Therefore, observation data is needed to guide the deterministic system model to assimilate the true state of the contaminant. In reality, limited observation data exists since it is quite expensive and laborious to collect groundwater observation data. In this study, 9 grid points out of 400 have been used to simulate the observation site data. Observations are available at coordinates $(5,5),(5,10),(5,15),(10,5),(10,10),(10,15),(15,5)$, $(15,10)$ and $(15,15)$ [Figure 1]. This method was first considered by Chang and Jin (2005), where four observation points were used to run the KF model for spatially-independent Gaussian conditions. In this study, both UKF and KF were simulated based on those 9 observation grid points. In the process of generating the true solution data, the observation data is extracted from the true solution at the 9 observation points, also adding Gaussian random errors having a standard deviation of $5 \%$ [Equation (7)].

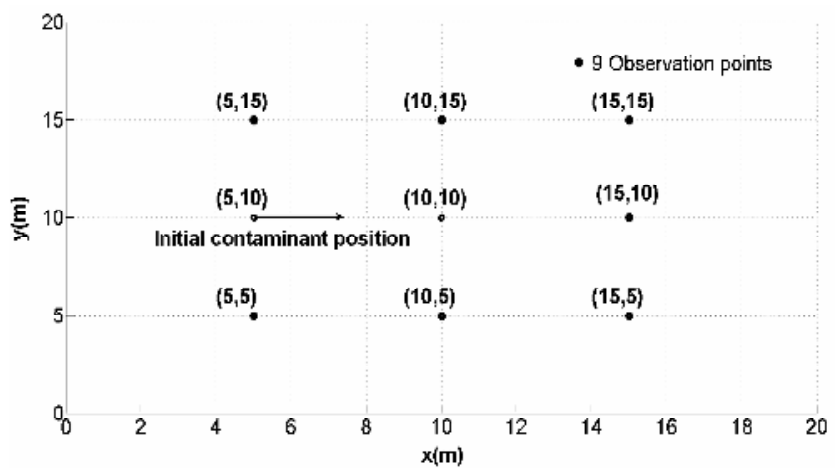

Figure 1. Initial contaminant and observation location in the model area.

\subsection{Examination of Models Scheme}

The effectiveness of the filters and deterministic model is determined by comparing the model predictions with the true solution. The Error Standard Deviation (ESD) indicates the average error in the predictions with respect to the true solution; it is used as the standard error parameter in this study.

ESD is defined as:

$$
\operatorname{ESD}(t)=\sqrt{\frac{1}{N-1} \sum\left[C^{P}(x, y, t)-C^{T}(x, y, t)\right]^{2}}
$$

where $\operatorname{ESD}(t)=\operatorname{error}(\mathrm{mg} / \mathrm{L})$ at time step $\mathrm{t} ; C^{P}(x, y, t)=$ predicted value of concentration at node (x,y) at time step t; $C^{T}(x$, $y, t)=$ true value of concentration at node $(x, y)$ at time step $t$; $n=$ total number of nodes.

\section{Results and Discussion}

MATLAB was used in this study to generate the algorithms for solving the FTCS, UKF, and KF models. The deter- ministic numerical model is the basis by which the state was found by utilizing the data assimilation model for UKF and KF. Equation (29) has been used for the comparison of the Error Standard Deviation (ESD) with the true solution. The average measurement error is calculated for all the time steps and spaces within the area under study. Figures 2 and 3 show the ESD of all the models used in this study. It was found that the filtering technique seems to have fewer errors than the deterministic model results. The error profile shows that the numerical scheme is the approach with the maximum errors at all-time steps. This is due to the approximation and assumptions made to the model which introduced a certain amount of error. Initially there is a small amount of error because the filtering process and the estimation by numerical process had not yet begun to indicate the error in prediction. The average prediction error in each time step is approximately $6,4.9$ and $3.1 \mathrm{mg} / \mathrm{l}$ in the numerical KF and UKF model results, respectively [Figure 2]. The average percentage reduction of prediction error for the UKF model calculated for all 50 time steps is approximately 49 and $37 \%$ when compared with the numerical and KF model, respectively [Figure 2]. In Figure 2, It is seen that after 50 time steps (10 days), UKF is $2.6 \mathrm{mg} / \mathrm{l}$ which is lower than both $4 \mathrm{mg} / \mathrm{l}$ from the $\mathrm{KF}$ and $5.5 \mathrm{mg} / \mathrm{l}$ from the numerical model.

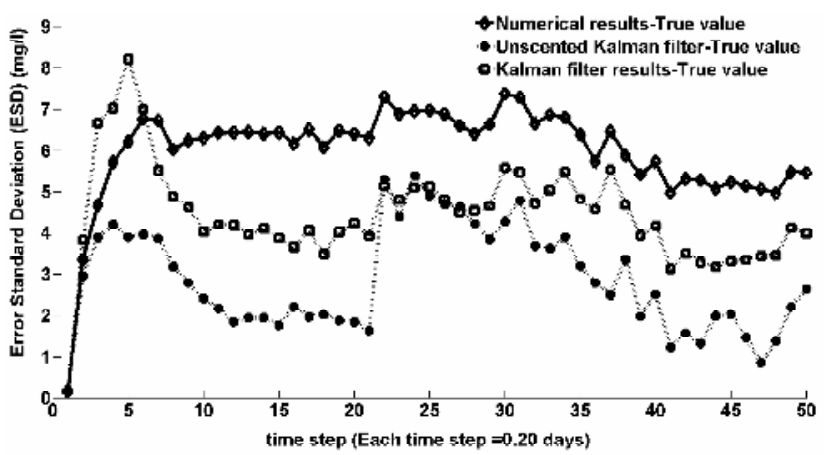

Figure 2. Error Standard Deviation (ESD) (mg/l) of numerical, KF and UKF models.

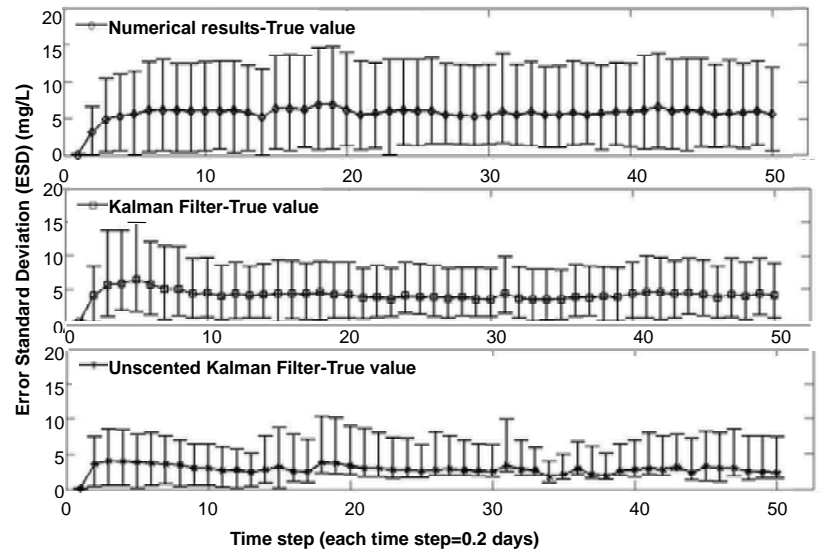

Figure 3. Several runs of Error Standard Deviation (ESD) of numerical, KF and UKF models. 
Several repeated runs were conducted and the results of each model were compared with the true solution [Figure 3]. The model results of the 5 repeated runs are shown by the error bar in Figure 3. The lower value of each time step in the error bar is the difference between the average and the lowest value of 5 runs. The upper value of each time step in the error bar is the summation of the average and the highest value of 5 runs. In the first several time steps, the ESDs are going up due to the divergence of the numerical scheme from the true stochastic field. Contaminant concentrations will decrease with time due to the dispersion and therefore the ESD is lower after 40 time steps [Figure 2].

The improved performance of the UKF compared to the $\mathrm{KF}$ is due to two factors: the increased time-update accuracy and the improved covariance accuracy. The covariance estimation can be quite different for the two filters as shown in Equations (6), (7), (20), (21), (24) and (25). In the KF process, noise covariance $\mathrm{Q}$ and measurement noise covariance $\mathrm{R}$ are symmetric, diagonal, and positive definite in nature. These $\mathrm{Q}$ and $\mathrm{R}$ are directly generated from the numerical results multiplied with their noises and then squared. But in the UKF model, these covariances are the weighted outer products of the transformed numerical values (Rambabu et al. 2008). To avoid the semi singular error covariance matrix in Equation (9) for the $\mathrm{KF}$ operation, a small positive entry is required rather than a zero matrix. These small positive entries are placed on the diagonal of a matrix. A badly scaled singular matrix can be formed if a bigger number is put to construct an initial error covariance matrix. But the UKF doesn't have this type of problem. Even a high initial error variance can have negligible effects on the prediction by the UKF (Rudolph and Eric 2001).

The predictions after 4 days from the numerical, KF and UKF models are shown in Figure 4. It is clearly indicated that the pollutant plume in the numerical model moves faster than the plume of the true solution due to the inaccurate $\mathrm{V}$ used in the prediction model when compared with the other models.

The KF and UKF data assimilation schemes depend on the generation of the observation data from the true solution model results to serve as a guide in estimating the state of the plume concentration. In this research, Gaussian system error with a standard deviation of $5 \%$ is injected into the simulated true solution to generate the measurement or observation data. For this reason, the nature of the spreading or dispersion of the contaminant plume of the KF and UKF data assimilation schemes and the true solution is similar [Figure 4]. The closeness of the contours with the true solution indicates the small deviation or prediction error from that model.

The prediction results after 6 days from the numerical, $\mathrm{KF}$ and UKF models are shown in Figure 5. The plume size increased with time in all the model contours. The contours predicted by the UKF and KF models coincide with the true solution more closely than those predicted by the numerical solution. As described earlier, ESD is used as the average measurement of the standard error between the true data and the model prediction in the entire space domain (400 nodes). It is mentionable here that to take care of the spatial sparseness of data, a sparse observation data pattern matrix is created. Here measurement sensitivity matrix, $\mathbf{H}$ is a $9 \times 400,0-1$ matrix. It has " 1 " in nine different observation sites and " 0 " in the remaining grid points.

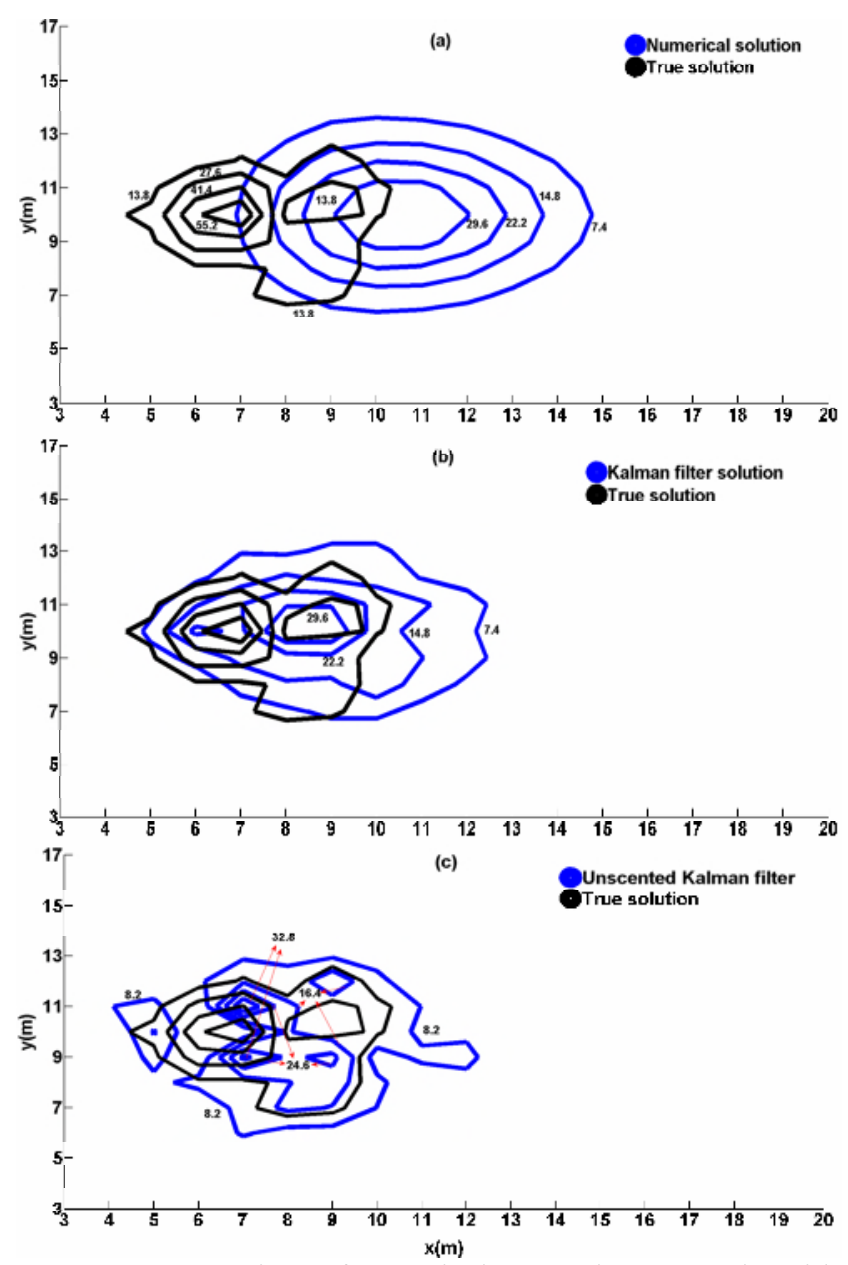

Figure 4. Comparison of numerical, KF and UKF results with the true solutions of the contaminant contour after 20 time steps (4 days).

The predictions after 10 days from the numerical, $\mathrm{KF}$ and UKF models are shown in Figure 6. In these contours, indications show that the UKF model is able to predict the irregular shapes of the natural stochastic system more closely than the KF and deterministic models do. The contours of the UKF results in Figures 4, 5 and 6 are relatively closer to the true value than the KF results and the numerical results. The performance of the UKF model is dependent on the proper weights it is assigned to the samples and choices of the tuning parameters. The sigma points chosen in Equations (12), (13) and (14) also play an important role in the accuracy of the prediction process. Performance of the UKF can also be dependent on the covariance's generation in Equations (20), (21) and (24). Figure 5 which represents time step 30, KF model provides the best contaminant concentration contour with the true solution when compared with the time step 20 and time step 50 in Figures 4 and 6 , respectively. 


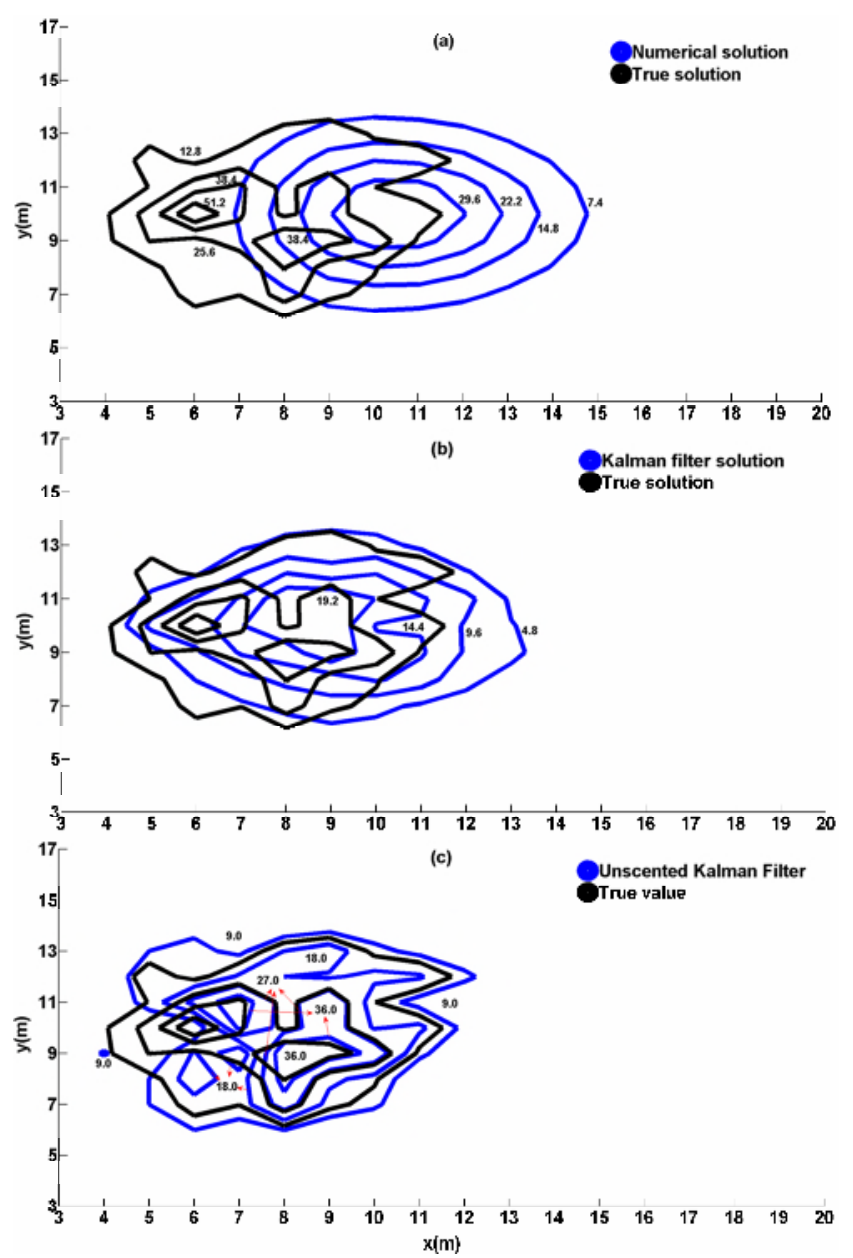

Figure 5. Comparison of numerical, KF and UKF results with the true solutions of the contaminant contour after 30 time steps (6 days).

The perfect bell shape pollutant contour line in all the numerical results in Figures 4, 5 and 6 represents the theoretical advection-dispersion transport process. The deviation between the numerical and true solution in Figure 6 was visibly clear. Deterministic numerical model approximation provides a relatively, smooth shape of the contaminant plume due to the random stochastic field was not introduced. The filtering adjusts the system model by the observation data and tries to project the optimal state. But the deterministic model relies only on the initial and boundary conditions, the assumed flow parameters, and its systems of equations to predict the state.

At time step 20 in the true solution contour, the contaminant concentrations are 55.2, 41.4, 27.6 and $13.8 \mathrm{mg} / \mathrm{l}$. At time step 50 in the true solution, the contaminant concentrations are $35.2,26.4,17.6$ and $8.8 \mathrm{mg} / 1$. These contours clearly represent that contaminant concentrations are decreasing with the propagation of time. After comparing the model contours in three figures, it is obvious that the stochastic model with the UKF data assimilation is more accurate than the KF data assimilation and the traditional numerical approach.



Figure 6. Comparison of numerical, KF and UKF results with the true solutions of the contaminant contour after 50 time steps (10 days).

In Figures 7 and 8 shows the contaminant concentration at four different grid points in the model area, in four different model schemes within the simulated period. In Figure 7, the grid points are $(6,10) ;(7,10) ;(8,10) ;(9,10)$ and in Figure 8 the grid points are $(10,10) ;(11,10) ;(12,10) ;(13,10)$. The initial pulse input position was $(5,10)$ [Fgiure 1]. Therefore, the highest pollutant concentration exists on these grid points in the model area. Four plots in each figure are done in the same scale. The contaminant concentration decreases in all the four model schemes within the simulated period in Figures 7 and 8 when the grid position is changing.

Smooth concentration distribution curves in Figures 7 and 8 show that stochastic random errors were not introduced in the deterministic numerical model. Numerical model results also show the deviated concentration at all time steps at all grid points in Figures 7 and 8. It is due to the approximation and assumptions made to the model which introduced a certain amount of error. It is visibly clear in Figures 7 and 8 that the UKF coincides with the true solution and gives the best result compared with the numerical and the KF model. 

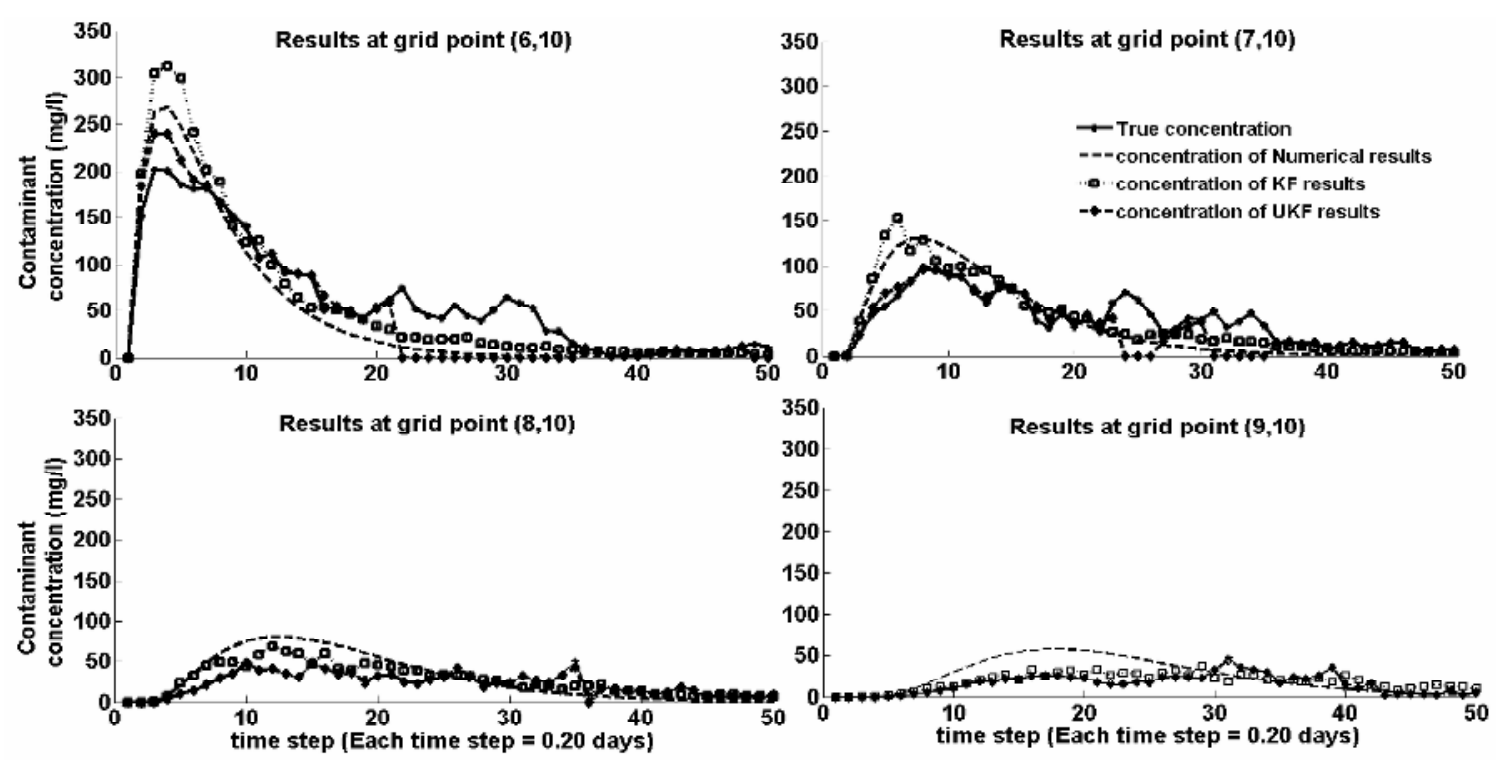

Figure 7. Concentration profiles at four different grid points in the different models.
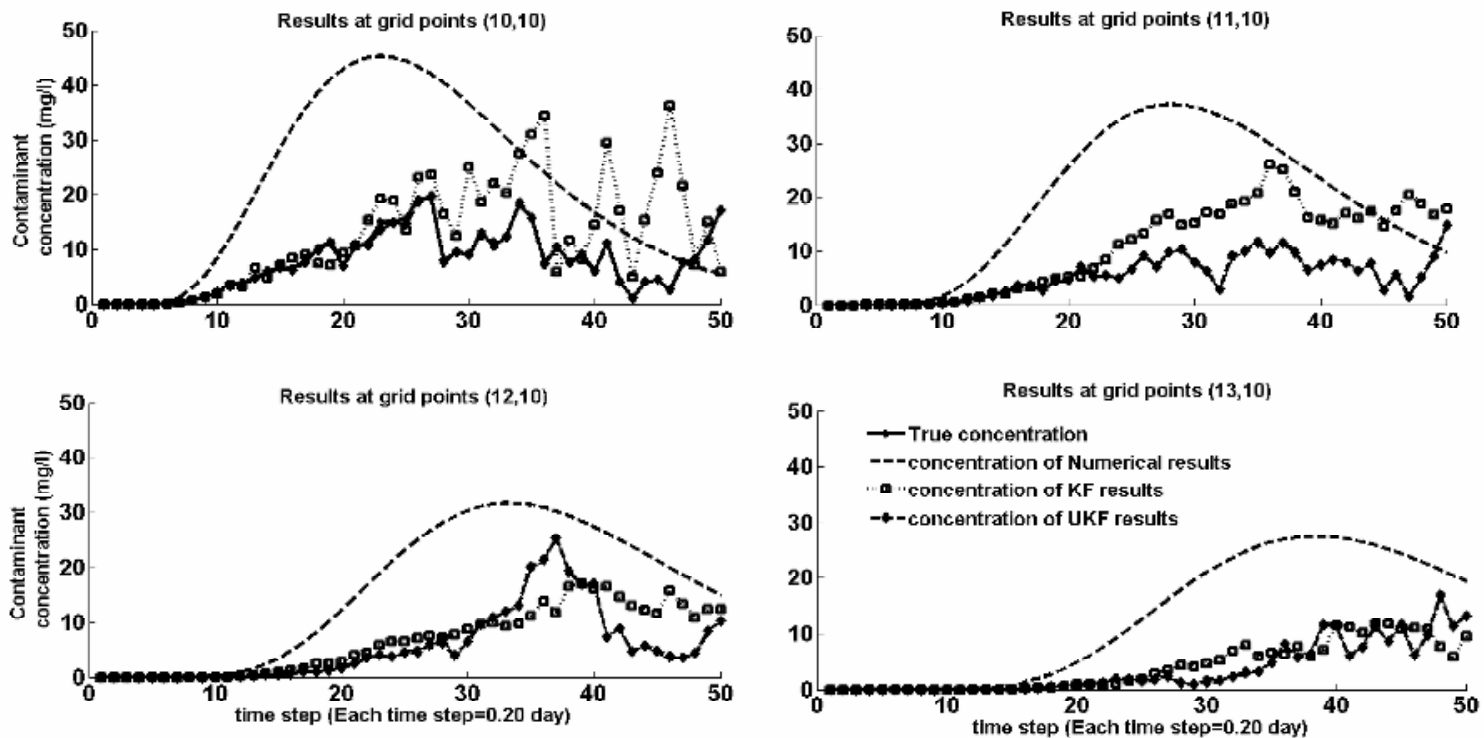

Figure 8. Concentration profiles at four different grid points in the different models.

\section{Conclusions}

The complex dynamic behavior of contaminant transport within groundwater environment systems has been studied using numerical methods. However, errors from the deterministic numerical method can bring unavoidable prediction deviations from the real world. Errors associated with the tradetional numerical method may include numerical errors from model mechanisms, time and space limits of numerical schemes, and boundary conditions. Using filtering techniques with the combination of the observation information into model dynamics should give a more accurate estimation result compared to that from the deterministic numerical method. This study introduced the use of the Unscented Kalman Filter (UKF) in a typical groundwater modeling application and compared the results with the Kalman Filter (KF) and the deterministic numerical method. The effectiveness of the proposed method was then evaluated by Error Standard Deviation (ESD). The KF and UKF schemes are both constructed using MATLAB routines and operated with the FTCS dynamics. The KF and UKF model simulation time was around seven minutes while the numerical results take an average of two minutes of running time on a $2.0 \mathrm{GHz} \mathrm{PC}$ with $3.0 \mathrm{~GB}$ RAM. The distinct difference in error for all the approaches is best seen when the ESD is determined. It was found that the UKF data assimilation method is able to provide good results in comparison with the deterministic numerical method and the KF method. With only nine observations out of a total 400 grid points in the model area, the UKF can reduce $6 \sim 75 \%$ and $2 \sim 52 \%$ of prediction errors when compared with the deterministic and 
KF results, respectively. With sparse observation data points as in the case of a pulse input subsurface contaminant transport, the UKF scheme can provide better prediction than the KF scheme and deterministic numerical model.

Acknowledgments. This work was sponsored by the Department of Energy Samuel Massie Chair of Excellence Program under Grant No. DF-FG01-94EW11425. The views and conclusions contained herein are those of the writers and should not be interpreted as necessarily representing the official policies or endorsements, either expressed or implied, of the funding agency.

\section{References}

Andrei, R., José, A.A.M. C. (2004). The unscented filter as an alternative to the EKF for nonlinear state estimation: a simulation case study. Computers \& Chemical Engineering, 28, 347-355. http:// dx.doi.org/10.1016/j.bbr.2011.03.031

Chan, P.T., and Govindaraju S.R. (2006). A stochastic-advective transport model for NAPL dissolution and degradation in nonuniform flows in porous media. J. Contam. Hydrol., 87, 253-276. http://dx.doi.org/10.1016/j.jconhyd.2006.05.010.

Chang, S.Y., and Jin, A. (2005). Kalman Filtering with regional noise to improve accuracy of contaminant transport models. J. Environ. Eng., 131(6),971-982. http://dx.doi.org/10.1061/(ASCE)0733-9372 (2005)131:6(971).

Cheryl, C.Q., and Juergen, H. (2008). Process monitoring and parameter estimation via Unscented Kalman Filtering. Journal of Loss Prevention in the Process Industries, 1-7.

Costa, P.J. (1994). Adaptive model architecture and Extended Kalman Bucy filters. IEEE Transp. Aerospecis Electron. Syst., 30, 525-533. $\mathrm{http}: / / d x . d o i . o r g / 10.1109 / 7.272275$.

Creveling, R.D., Philip E.G., and Henry, D.I.A. (2008). State and parameter estimation in nonlinear systems as an optimal tracking problem. Phys. Lett. A, 372, 2640-2644. http://dx.doi.org/10.1016 /j.physleta.2007.12.051.

Goovaerts, P. (1999). Geostatistics in soil science: state-of-the-art and perspectives. Geoderma, 89, 1-45. http://dx.doi.org/10.1016/S0016 -7061(98)00078-0.

Gove, J.H., and Hollinger, D.Y. (2006). Application of a dual Unscented Kalman Filter for simultaneous state and parameter estimation in problems of surface-atmosphere exchange. J. Geophys. Res., 111, D08S07. http://dx.doi.org/10.1029/2005JD 006021.

Grewal, M.S., and Andrews, A.P. (1993). Kalman Filtering: Theory and practice. Englewood Cliffs, NJ: Prentice Hall.

Hamed, M.M., Bedient, B.P., and Conte P.J. (1996). Numerical stochastic analysis of groundwater contaminant transport and plume containment. J. Contam. Hydrol., 24, 1-24. http://dx.doi.org /10.1016/0169-7722(96)00007-1.

Heuvelink, G.B.M., and Webster, R. (2001). Modeling soil variation: past, present, and future. Geoderma, 100, 269-301. http://dx.doi. org/10.1016/S0016-7061(01)00025-8.

Hu, X.B., Mark, M.M., Warren, B., David, W.H., He, C., Li X., and Guo, L. (2009). Examining the influence of heterogeneous porosity fields on conservative solute transport. J. Contam. Hydrol., 108, 77-88. http://dx.doi.org/10.1016/j.jconhyd.2009.06.001.

Jin, A. (1996). An optimal estimation scheme for subsurface contaminant transport model using Kalman-Bucy filter. MS thesis, North Carolina A\&T State University, Greensboro, N.C.

Julier, S.J., and Uhlmann, J.K. (1997). A new extension of the Kalman filter to nonlinear systems. The Proceedings of Aerosense: The 11th International Symposium on Aerospace/Defense Sensing, Simulation and Controls, 182-193.

Julier, S.J., and Uhlmann, J.K. (2004). Unscented Filtering and nonlinear estimation. Proceedings of the IEEE Aerospace and Electronic Systems, 92 (3), 410-422.
Kim, J.M., and Parizek R.R. (1999). Three-dimensional finite element modeling for consolidation due to groundwater withdrawal in a desaturating anisotropic aquifer system. Int. J. Numer. Anal. Meth. Geomatics, 23, 549-571.http://dx.doi.org/10.1002/(SICI) 1096-9853 (199905)23:6<549::AID-NAG983>3.0.CO;2-Y.

Li, H., and Zhang, K. (2010). Development of a fuzzy-stochastic nonlinear model to incorporate aleatoric and epistemic uncertainty. J. Contam. Hydrol., 111, 1-12. http://dx.doi.org/10.1016/j.jconhyd. 2009.10.004

Magnus, N., Niels P.K., and Ole Ravn. (2000). New developments in state estimation for nonlinear systems. J. Automatica, 36, 1627 1638. http://dx.doi.org/10.1016/S0005-1098(00)00089-3.

Parkin, G., Birkinshaw, S.J., Younger, P. L., Rao, Z., and Kirk, S. (2007). A numerical modeling and neural network approach to estimate the impact of groundwater abstractions on river flows. $J$. Hydrol., 339 (1-2), 15-28. http://dx.doi.org/10.1016/j.jhydrol.2007. 01.041 .

Pham, D.T. (2001). Stochastic methods for sequential data assimilation in strongly nonlinear systems. Mon. Weather Rev., 129 (5), 1194-1207. http://dx.doi.org/10.1175/1520-0493(2001)129<1194: SMFSDA $>2.0 . \mathrm{CO} ; 2$.

Pastres, R., Ciavatta, S., and Solidoro, C. (2003). The Extended Kalman Filter (EKF) as a tool for the assimilation of high frequency water quality data. Ecol. Model., 170, 227-235. http:// dx.doi.org/10.1016/S0304-3800 (03)00230-8.

Rambabu, K., Bjarne F., and Lars I. (2008). Applying the Unscented Kalman Filter for nonlinear state estimation. J. Process Control, 18, 753-768. http://dx.doi.org/10.1016/j.jprocont.2007.11.004.

Ren, L., and Zhang R. (1999). Hybrid Laplace transform finite element method for solving the convection-dispersion problem. Adv. Water Resour., 23, 229-237. http://dx.doi.org/10.1016/S0309 -1708(99)00013-5.

Rudolph, V.M., and Eric A.W. (2001). The square-root Unscented Kalman Filter for state and parameter-estimation. IEEE Int. Conf., 6, 3461-3464.

Saad, G.A. (2007). Stochastic data assimilation with application to multi-phase flow and health monitoring problems. Doctoral dissertation, University of Southern California, Los Angeles.

Schreider, S.Y., Yong, P.C., and Jakeman, A.J. (2001). An application of the Kalman Filtering technique for stream flow forecasting in the upper Murray basin. Math. Comput. Model., 33, 733-743. http://dx.doi.org/10.1016/S0895-7177(00)00276-4.

Simon, J., Jeffrey U., and Hugh F.D. (2000). A New Method for the Nonlinear Transformation of Means and Covariances in Filters and Estimators. IEEE Transaction on Automatic Control, 45(3).

Van, G., Martinus, T., and Sudicky, E.A. (1999). Recent advances in vadose zone flow and transport modeling. In: M.B. Parlange and J.W. Hopmans (eds.), Vadose zone hydrology: cutting across disciplines, 155-193, Oxford University Press, New York.

Wan, E.A., and Rudolph, V.M. (2000). The Unscented Kalman Filter for nonlinear estimation. In Proceedings of Symposium 2000 on Adaptive Systems for Signal Processing, Communication, and Control (AS-SPCC). Lake Louise, Alberta, Canada: IEEE. 153 $-158$.

Zhang, K., Achari, G., and Li, H. (2009). A comparison of numerical solutions of partial differential equations with probabilistic and possibilistic parameters for the quantification of uncertainty in subsurface solute transport. J. Contam. Hydrol., 110, 45-59. http:// dx.doi.org/10.1016/j.jconhyd.2009.08.005.

Zheng, C., and Bennett, G.D. (2002). Applied Contaminant Transport Modeling. Second Edition, 621, John Wiley \& Sons, New York.

Zou, S., and Parr, A. (1995). Optimal Estimation of two-dimensional Contaminant transport. Ground Water, 33, 319-325. http://dx.doi. org/10.1111/j.1745-6584.1995.tb00287.x. 